\title{
Finite Element Analysis of Bi-Modulus Ceramic Structure
}

\author{
Du Ling \\ Transportation Equipment and Ocean Engineering \\ College \\ Dalian Maritime University \\ Dalian, China \\ e-mail: yige0729@126.com
}

\author{
Li Fanchun \\ Transportation Equipment and Ocean Engineering \\ College \\ Dalian Maritime University \\ Dalian, China \\ e-mail: lee_fc@126.com
}

\begin{abstract}
By judging positive and negative of stress balls tensor of ceramic beam structure, to determine tension and compression elastic modulus and the position of the neutral layer of the beam structure, the structural analysis of the ceramic beam could be done. The results showed that, there was a great error value between the first principal stress calculated by bi-modulus and by single-modulus. So did other stresses. The error value completely exceeded the allowable range of engineering. Only by defining the bimodulus, structural optimization analysis of ceramic materials could be accurately carried out. In this paper, the method is presented by judging positive and negative of stress balls tensor to determine tension and compression elastic modulus of the structure. Basing on Ansys, analysis modules of the materials of different modulus of elasticity in tension and compression have been developed. This method has relatively small calculation, wide range of applications and can even be applied to large, complex ceramic structures. The method for the optimization of the structure of the ceramic material has a very important practical significance.
\end{abstract}

Keywords-Ceramic beam; stress balls tensor; bi-modulus; single-modulus

\section{INTRODUCTION}

When the spacecraft enters the atmosphere in hypersonic speeds, it would be a strong aerodynamic heating. The outer surface of the spacecraft is surrounded by high-temperature air, and thermal protection systems (TPS $)^{[1,2]}$ must be installed. However, ultra-high temperature ceramic materials with good hightemperature mechanical properties, is one of the candidate materials of scramjet-powered aircraft leading edge components. Because scramjet engine service environment is very bad, requirements of hot structural materials (such as the leading edge of the tip wedge) on the high temperature mechanical performance are harsh. Hypersonic aerodynamic heating process cannot be ignored, which results in a large temperature gradient ${ }^{[3]}$ inside the material and thermal stress ${ }^{[4-8]}$ can significantly reduce the cycle life of the member and even leads to the stresses exceeding the flexural strength and fracture. Therefore, optimization of the structure of the thermal protection system $^{[9]}$ is imperative.

Wind tunnel ground simulation is the most common method of materials assessment, but there are costly, energy consumption and other issues. So materials design and optimization of the structural member are very difficult only by wind tunnel simulation. On the basis of the existing experimental studies, establishing a reasonable mathematical model and using numerical methods for simulating aerodynamic heating process of leading edge components of ultra-high temperature ceramic materials and the thermal stress distribution, this will not only help deepen understanding of the service process and the failure mechanism of ultra-hightemperature ceramic materials, and can shorten the cycle of material design and structural optimization, which has a very important leading significance of research on the leading edge components of ultra-high temperature ceramic materials used in scramjet engines. However, remarkable characteristics of the ceramic materials in tension and compression modulus of elasticity are quite different, which has attracted significant attention in the process of performing structural analysis.

$\mathrm{Wu}$ Xiao et $\mathrm{al}^{[10]}$ studied that the plane stress and displacement problem of a curved beam with difference elastic moduli in tension and compression. Meanwhile the stress and displacement formulas were derived. Then the calculation results were compared with those obtained by the finite element method, the reliability of the stress and displacement formulae for a curved beam with difference elastic moduli in tension and compression were verified. Ye Zhiming et $\mathrm{al}^{[11]}$ obtained the analytical and finite element solutions of cantilever beam problems. The errors between analytical and finite element solutions are discussed in details. Wu Xiao et al ${ }^{[12]}$ studied that the deformation of thick wall spherical shell of bi-modulus materials under uniform pressure with elastic theory. And stress formula for thick wall spherical shell was derived, with which the mohr strength theory the thickness of wall was calculated.

In summary, the different elastic modulus theory has caused widespread concern, and analytical solutions of simple structure, the finite element solutions can all be solved, which has a great contribution of impact of bimodulus method in the project. But for some large complex structures (such as assembly), the calculated amount is too large, the results are not necessarily accurate. Moreover, the existing software is not able to define bi-modulus and solve computing problems. This paper presents a method with relatively small amount of 
computation for a wide range. That is by judging the positive or negative stress ball tensor to determine the elastic modulus in tension or compression of the beam structure. And based on Ansys, the analysis modules of bi-modulus materials have been developed. Ceramic beam, for example, comparing with single-modulus and bimodulus calculations, analyzing errors, from the comparison of the data results, bi-modulus used in structural analysis of engineering is very important.

\section{CAlculation MethoD}

For the members subjected to the force, the stress state of each point can be symmetrical with a secondorder tensor, i.e.

$$
\sigma_{i j}=\left(\begin{array}{ccc}
\sigma_{x} & \tau_{x y} & \tau_{x z} \\
\tau_{y x} & \sigma_{y} & \tau_{y z} \\
\tau_{z x} & \tau_{z y} & \sigma_{z}
\end{array}\right) .
$$

Where, $\sigma_{\mathrm{x}}$ is the $\mathrm{x}$-axis normal stress. $\sigma_{\mathrm{y}}$ is the y-axis normal stress. $\sigma_{\mathrm{z}}$ is the $\mathrm{z}$-axis normal stress. $\tau_{\mathrm{xy}}$ is the $\mathrm{y}$ axis shear stress and $\tau_{\mathrm{xz}}$ is the $\mathrm{z}$-axis shear stress, which are on the x-plane. $\tau_{\mathrm{yx}}$ is the $\mathrm{x}$-axis shear stress and $\tau_{\mathrm{yz}}$ is the $\mathrm{z}$-axis shear stress, which are on the $y$-plane. $\mathrm{T}_{\mathrm{zx}}$ is the $\mathrm{x}$-axis shear stress and $\tau_{\mathrm{zy}}$ is the $\mathrm{y}$-axis shear stress, which are on the z-plane.

$$
\text { Assume } \begin{aligned}
\sigma_{\mathrm{x}} & =\sigma_{\mathrm{x}}^{\prime}+\sigma_{\mathrm{m}}, \\
\sigma_{\mathrm{y}} & =\sigma_{\mathrm{y}}^{\prime}+\sigma_{\mathrm{m}}, \\
\sigma_{\mathrm{z}} & =\sigma_{\mathrm{z}}^{\prime}+\sigma_{\mathrm{m}} .
\end{aligned}
$$

Then, depending on the tensor nature, the stress tensor can be decomposed into the sum of two tensors.

$$
\begin{gathered}
\sigma_{i j}=\left(\begin{array}{ccc}
\sigma_{x} & \tau_{x y} & \tau_{x z} \\
\tau_{y x} & \sigma_{y} & \tau_{y z} \\
\tau_{z x} & \tau_{z y} & \sigma_{z}
\end{array}\right)= \\
\left(\begin{array}{ccc}
\sigma_{x}^{\prime}+\sigma_{m} & \tau_{x y} & \tau_{x z} \\
\tau_{y x} & \sigma_{y}^{\prime}+\sigma_{m} & \tau_{y z} \\
\tau_{z x} & \tau_{z y} & \sigma_{z}^{\prime}+\sigma_{m}
\end{array}\right)= \\
\left(\begin{array}{ccc}
\sigma_{x}^{\prime} & \tau_{x y} & \tau_{x z} \\
\tau_{y x} & \sigma_{y}^{\prime} & \tau_{y z} \\
\tau_{z x} & \tau_{z y} & \sigma_{z}^{\prime}
\end{array}\right)+\left(\begin{array}{ccc}
\sigma_{m} & 0 & 0 \\
0 & \sigma_{m} & 0 \\
0 & 0 & \sigma_{m}
\end{array}\right) . \\
\text { i.e. } \quad \sigma_{\mathrm{ij}}=\sigma_{\mathrm{ij}}^{\prime}+\delta_{\mathrm{ij}} \sigma_{\mathrm{m}} .
\end{gathered}
$$

Wherein the stress tensor $\sigma_{\mathrm{ij}}^{\prime}$ is called partial stress tensor, it only causes changes in body shape and plastic deformation of materials is caused by it. The latter $\delta_{\mathrm{ij}} \sigma_{\mathrm{m}}$ is called the stress balls tensor, and it is the main direction in any direction. Features of ball stress state are not on any shear stress in the tangent plane. It cannot change the shape of an object, and can only produce a volume change. And the principal stresses are average stress:

$$
\sigma_{\mathrm{m}}=\frac{1}{3}\left(\sigma_{1}+\sigma_{2}+\sigma_{3}\right)=\frac{1}{3} J_{1} .
$$

Where, $\sigma_{1}, \sigma_{2}, \sigma_{3}$ is the first, second, third principal stress. And $\mathrm{J}_{1}$ is one of three invariants of the stress tensor. So, $\sigma_{\mathrm{m}}$ is an invariant. It has nothing to do with the coordinates taken. For a determining state of stress, it is a single value. According to the characteristics of the stress balls tensor, defining tension and compression elasticity modulus, suppose

$$
\sigma_{\mathrm{m}}=\sigma_{1}+\sigma_{2}+\sigma_{3}>0 .
$$

Volume increasing, the tensile deformation occurs, and this point is defined as the elasticity modulus. If

$$
\sigma_{\mathrm{m}}=\sigma_{1}+\sigma_{2}+\sigma_{3}<0,
$$

volume decreasing, compression occurs, this point is defined as the compression elasticity modulus. When the tension and compression elasticity modulus of all the points defined, the whole structure can complete the definition.

\section{THE FLOW CHART}

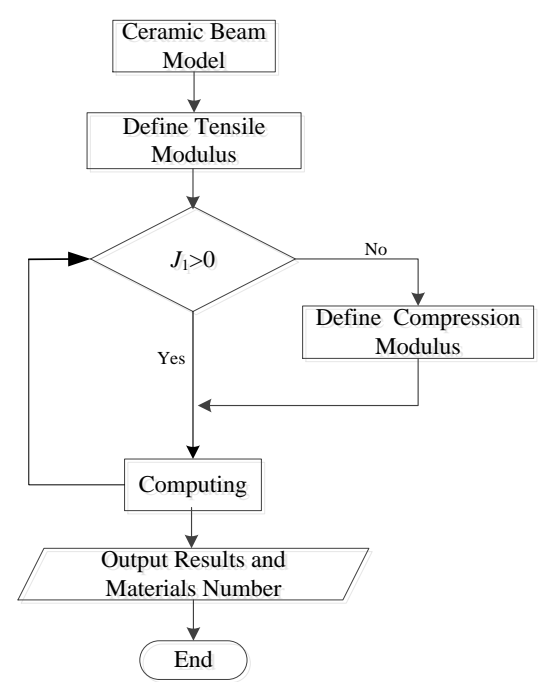

\section{CERAMIC BEAM STRUCTURE ANALYSES}

Ceramic beam structure, for example, the modulus of elasticity in tension and compression ratio of $1 / 2$, the establishment of a ceramic beam Ansys model is done. Aside fixed end, B-side free end, the unit displacement is applied in Fig. 1.

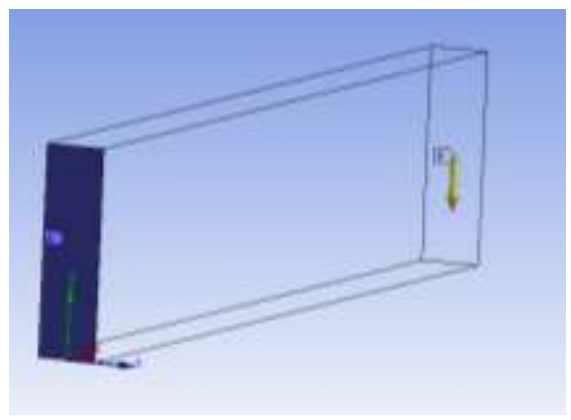

Figure. 1 the ceramic beam

\section{A. Single-modulus ceramic beam structure analysis}

Applying the theory of classical mechanics, i.e. the same modulus, according to the same tension and 
compression modulus of elasticity for calculating, the distribution of the first principal stress $\sigma_{1}$ and the third principal stress $\sigma_{3}$, which is along $\mathrm{y}$-axis at the fixed end of the single-modulus ceramic beam, is shown in Fig. 2.

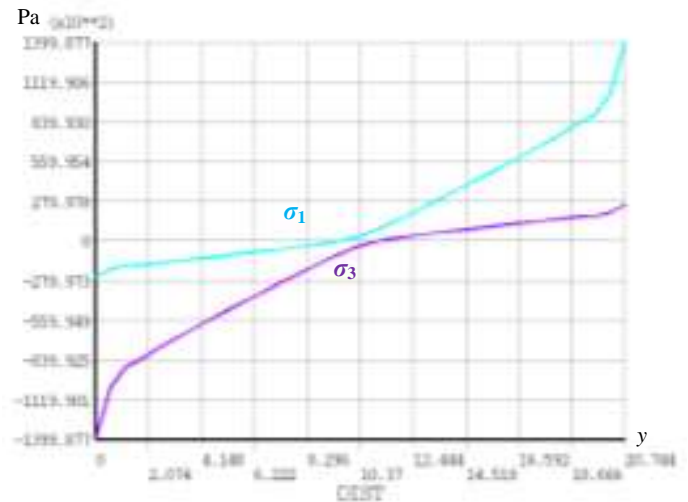

Figure. 2 the first principal stress $\sigma_{1}$ and the third principal stress $\sigma_{3}$ distribution along the y-axis of the single-modulus ceramic beam at the fixed end

In accordance with the same tension and compression modulus of elasticity for calculating, the distribution of the x-axis normal stress $\sigma_{\mathrm{x}}$, which is along $y$-axis at the fixed end of the single-modulus ceramic beam, is shown in Fig. 3.

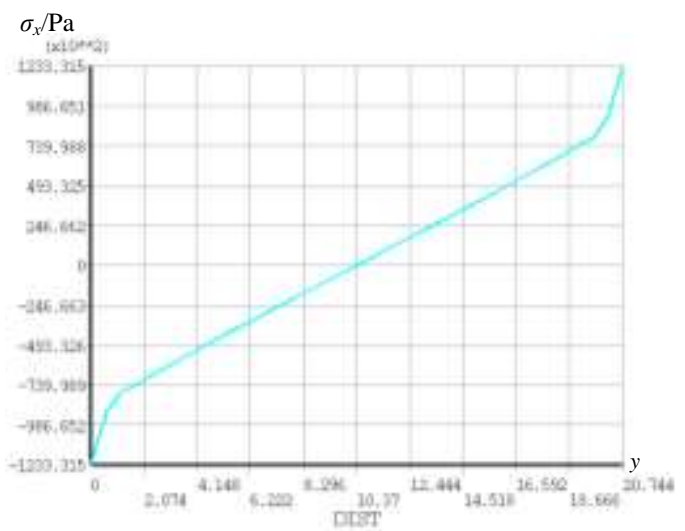

Figure. 3 the distribution along the y-axis path of normal stress $\sigma_{\mathrm{x}}$ along the x-axis of the single -modulus ceramic beam at the fixed end

As can be seen from Figure 3, the absolute value of the normal stress along $\mathrm{x}$-axis of the upper and lower surfaces at the beam end is equal. This is the characteristics of the beam whose tensile modulus of elasticity is equal to compressive modulus.

After calculation, the distribution of the Mises stress $\sigma_{\text {mises }}$, which is along y-axis at the fixed end of the singlemodulus ceramic beam, is shown in Fig. 4.

It can be seen that, the curve is symmetrical about the neutral layer and the value of the Mises stress of the upper and lower surfaces at the beam end is equal.

$\sigma_{\text {mises }} / \mathrm{Pa}$

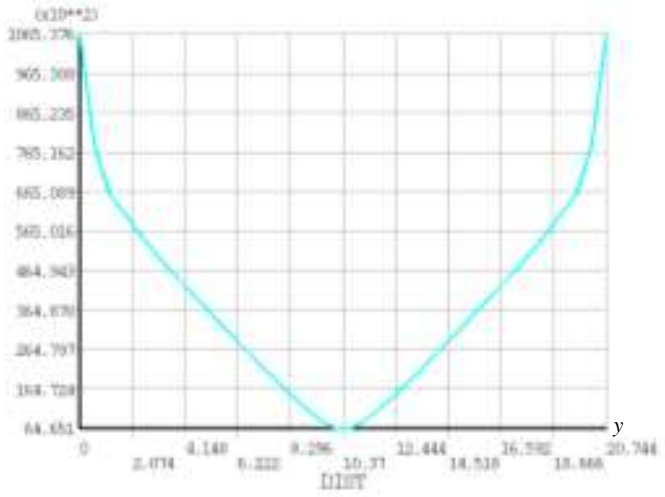

Figure. 4 the Mises stress $\sigma_{\text {mises }}$ distribution along the $y$-axis path of the single-modulus ceramic beam at the fixed end

After calculating, the distributions of the y-axis shear stress $\tau_{\mathrm{xy}}$ and the z-axis shear stress $\tau_{\mathrm{xz}}$, which is on the $\mathrm{x}$ plane at the fixed end of the single-modulus ceramic beam, are shown in Fig. 5 and Fig. 6.

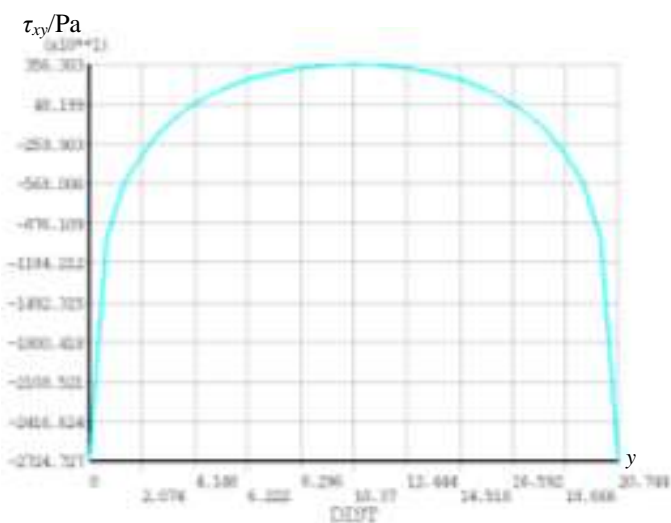

Figure. 5 the distribution of shear stress $\tau_{\mathrm{xy}}$ at the fixed end of single-modulus ceramic beam

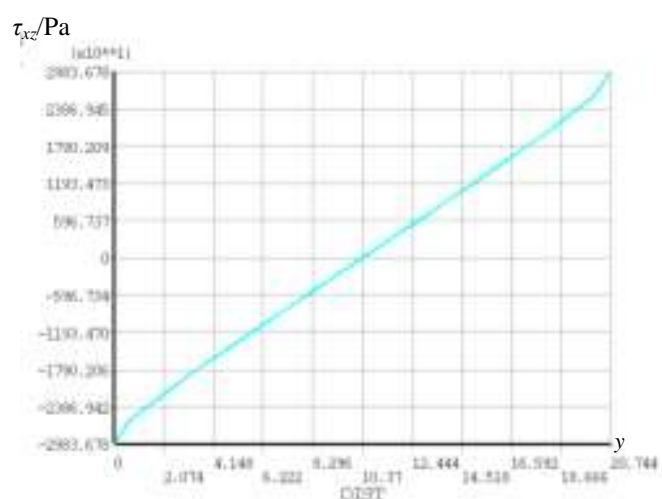

Figure. 6 the distribution of shear stress $\tau_{\mathrm{xz}}$ at the fixed end of single-modulus ceramic beam

B. Bi-modulus ceramic beam structure analysis

In fact, the ceramic beam modulus of elasticity in tension and compression ratio of $1 / 2$, according to the method by the judgment that the stress balls tensor is positive or negative to determine the modulus of elasticity in tension or compression, suppose

$$
\sigma_{\mathrm{m}}=\sigma_{1}+\sigma_{2}+\sigma_{3}>0,
$$

The point is the definition of a tensile elastic modulus.

$$
\sigma_{\mathrm{m}}=\sigma_{1}+\sigma_{2}+\sigma_{3}<0,
$$


This point is defined compression modulus. The elastic modulus in tension and compression of each point is determined. The location of the neutral layer is also determined, as shown in Fig. 7.

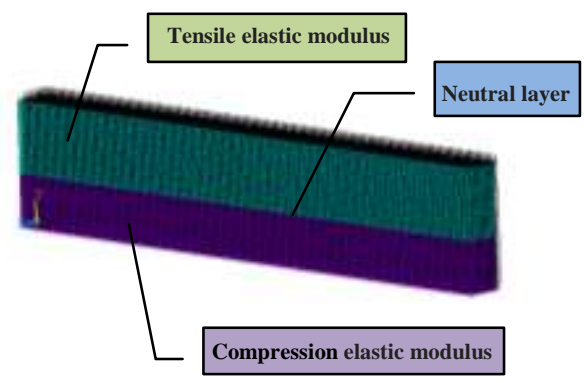

Figure. 7 the neutral layer of the ceramic beam

After determining the neutral layer, by calculation, the distribution of the first principal stress $\sigma_{1}$ and the third principal stress $\sigma_{3}$, which is along y-axis at the fixed end of the bi-modulus ceramic beam, is shown in Fig. 8 .

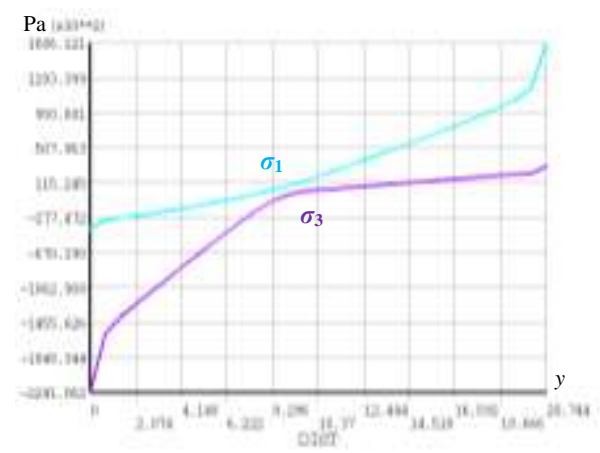

Figure. 8 the first principal stress $\sigma_{1}$ and the third principal stress $\sigma_{3}$ distribution along the y-axis path of the bi-modulus ceramic beam at the fixed end

By calculation, The distribution of the $\mathrm{x}$-axis normal stress $\sigma_{\mathrm{x}}$, which is along y-axis at the fixed end of the bimodulus ceramic beam, is shown in Fig. 9.

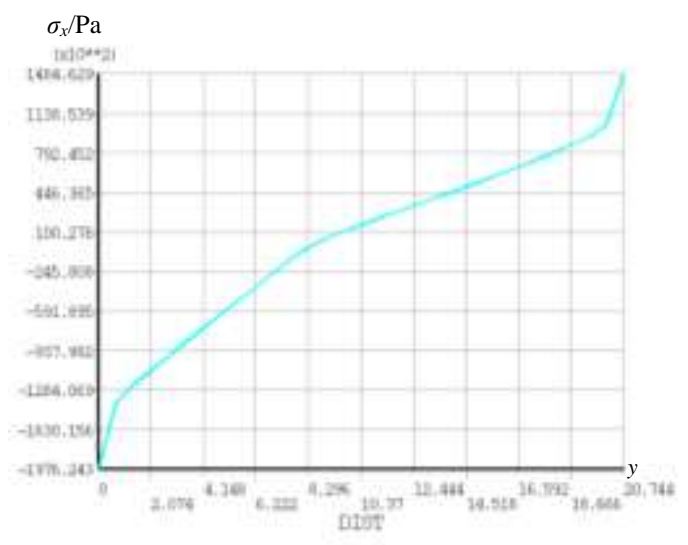

Figure. 9 the distribution along the y-axis path of normal stress $\sigma_{\mathrm{x}}$ along the x-axis of the b -modulus ceramic beam at the fixed end
The distribution of the Mises stress $\sigma_{\text {mises }}$, which is along y-axis at the fixed end of the bi-modulus ceramic beam, is shown in Fig. 10.

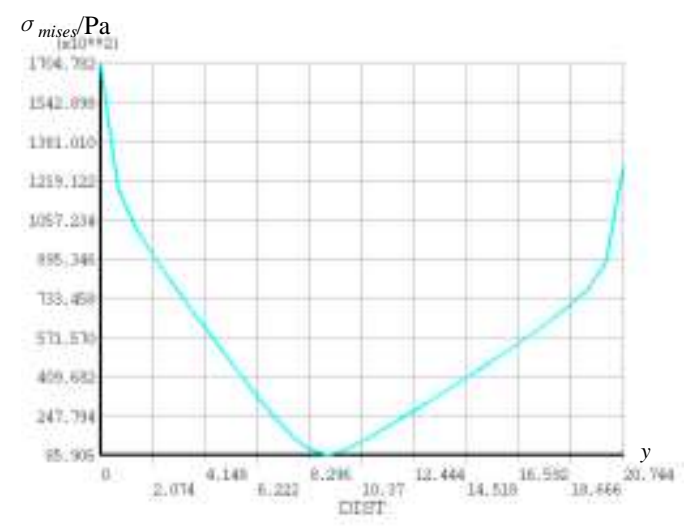

Figure. 10 the Mises stress $\sigma_{\text {mises }}$ distribution along the $y$-axis path of the bi-modulus ceramic beam at the fixed end

After calculating, the distributions of the $y$-axis shear stress $\tau_{\mathrm{xy}}$ and the $\mathrm{z}$-axis shear stress c, which is on the $\mathrm{x}$ plane at the fixed end of the bi-modulus ceramic beam are shown in Fig. 11 and Fig. 12.

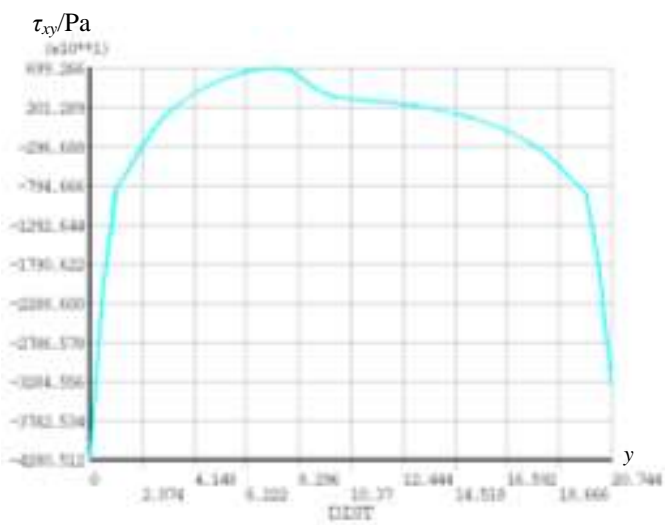

Figure. 11 the distribution of shear stress $\tau_{\mathrm{xy}}$ at the fixed end of bi-modulus ceramic beam

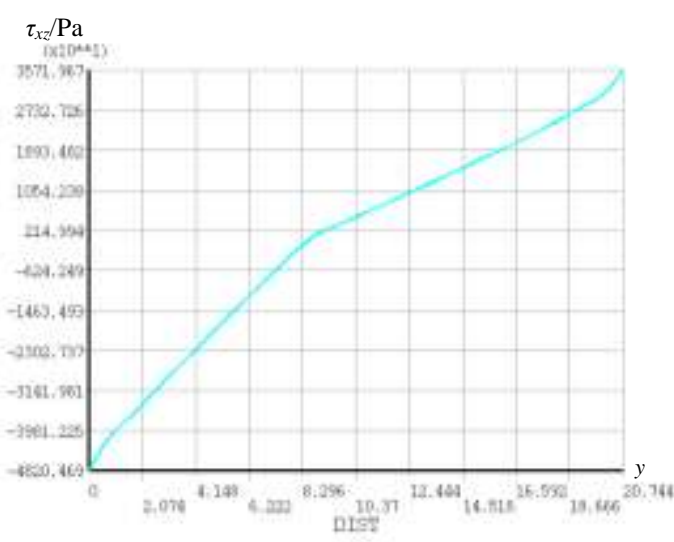

Figure. 12 the distribution of shear stress $\tau_{\mathrm{xz}}$ at the fixed end of bi-modulus ceramic beam

\section{ERROR ANALYSIS}

Write the maximum of the first principal stress $\sigma_{1}$, the third principal stress $\sigma_{3}$, the X-axis normal stress $\sigma_{\mathrm{x}}$, 
Mises stress $\sigma_{\text {mises }}$ and the y-axis shear stress $\tau_{\mathrm{xy}}$ and the zaxis shear stress $\tau_{\mathrm{xz}}$ on the x-plane along y-axis at the fixed end of single-modulus and bi-modulus ceramic beam in Table 1.

Comparing with the differences of the stress maximum at the fixed end of the ceramic beam by the way of classical mechanics and judging the stress balls tensor, the relative errors calculated are individually recorded in Table 1.

TABLE 1 COMPARISON BETWEEN SINGLE-MODULUS AND BI-MODULUS

\begin{tabular}{|c|c|c|c|}
\hline Position & $\begin{array}{c}\text { Maximum of } \\
\text { single-modulus } \\
(\boldsymbol{P a})\end{array}$ & $\begin{array}{c}\text { Maximum of } \\
\text { bi-modulus } \\
(\boldsymbol{P a})\end{array}$ & Error(\%) \\
\hline fixed end & $\sigma_{1}=139987$ & $\sigma_{1}=168612.1$ & 20.45 \\
\hline fixed end & $\sigma_{3}=139987.7$ & $\sigma_{3}=224106.2$ & 60.09 \\
\hline fixed end & $\sigma_{x}=123331.5$ & $\sigma_{x}=197624.3$ & 60.24 \\
\hline fixed end & $\sigma_{\text {mises }}=106537.6$ & $\sigma_{\text {mises }}=170478.2$ & 60.02 \\
\hline fixed end & $\tau_{x y}=27247.27$ & $\tau_{x y}=42805.12$ & 57.10 \\
\hline fixed end & $\tau_{x z}=29836.78$ & $\tau_{x z}=48204.69$ & 61.56 \\
\hline
\end{tabular}

\section{CONCLUSION}

By analyzing the error data in Table 1 , the errors of the first principal stress $\sigma_{1}$, the third principal stress $\sigma_{3}$, the x-axis normal stress $\sigma_{\mathrm{x}}$, Mises stress $\sigma_{\text {mises }}$ along y-axis at the fixed end between the single-modulus and bi-modulus ceramic beam are large. The errors of the $\mathrm{y}$-axis shear stress $\tau_{\mathrm{xy}}$ and the z-axis shear stress $\tau_{\mathrm{xz}}$ on the x-plane along $\mathrm{y}$-axis at the fixed end between the single-modulus and bi-modulus ceramic beam are large, too. They have exceeded the allowable error range of engineering. Especially the error of the $\mathrm{z}$-axis shear stress on the $\mathrm{x}$ plane between the single-modulus and bi-modulus ceramic beam is close to $62 \%$.

Thus, the stress calculated by the way of singlemodulus for the structural optimization of ceramic materials doesn't have practical significance. Only by defining the bi-modulus, the structural optimization of ceramic materials can be accurately done. Therefore, this paper presents the method that can judge tension or compression modulus of elasticity by judging the stress balls tensor whether positive or negative. This method has less computation for a wide range and even can be applied to large, complex ceramic structures. It has a very important practical significance for the optimization of structures of ceramic materials.

\section{REFERENCES}

[1] Qin Qiang, Jiang Junliang, Radiant thermal protection of TPS experimental study on the effectiveness. Journal of Sichuan Ordnance, 32(5), pp. 69-71, 2011.

[2] Yang Yazheng, Yang Jialing, Fang Daining, Progress of hypersonic vehicle thermal protection materials and structures.

[3] Applied Mathematics and Mechanics, 29(1), pp.47-56, 2008.

[4] Hao Jiguang, Jiang Yi, Liu Qi, Numerical Simulation on Aerodynamic Heating Process of Certain Missile s Head Using a Method of Fluid-solid Coupled. MISSILES AND GUIDANCE, 26(4), pp.230-232, 2006.

[5] Lin Xiaoxuan, Wu Haitang, Wei Xi, et al., Numerical Simulation on Aerodynamic Heating and Thermal Stress of ZrB2-SiC Leading Edge. Process Engineering,10(3), pp.417- 423, 2010.

[6] Liu Lei, Geng Xiangren, He Lixin, et al., Study on the thermal response/thermal stress for TPS with honeycomb. Chinese Engineering Thermo physics 2010 Conference on heat and mass transfer Conference Proceedings, Shanghai, 2011.

[7] Geng Xiangren, Gui Yewei, Xu Qingxin, et al., Calculation of thermal stress of thermal structure about a variety of materials .Chinese Engineering Thermo physics 2004 Heat and Mass Transfer Conference Proceedings (Volume 2), Shanghai, pp.1241-1245, 2005.

[8] Yi Long, Sun Qin, Peng Yun, Research of the analysis method of aerodynamic heating stress of a compisite cone. Journal of Mechanical Strength, 29(4), pp.686-690, 2007.

[9] Wang Zhangiian, Gui Yewei, Geng Xiangren, Transient thermal response and thermal stress calculation study about the structure similar to leading edge. Chinese Engineering Thermophysics 2002 Conference on heat and mass transfer Conference Proceedings, Shanghai, pp.785-788, 2003.

[10] Ma Zhonghui, Sun Qin, Wang Xiaojun, et al. Thermal model study of RLV ceramic thermal protection system. Missiles and Space Vehicles, (3), pp.19-24, 2003.

[11] Wu Xiao, Yang Lijun. The Elastic Theory Solution for Curved Beam With Difference Elastic Modulus in Tension and Compression. ENGINEERING MECHANICS, 30(1),pp.76-80, 2013.

[12] Ye Zhiming, Yao Wenjuan. Analytical and Finite Element Solutions of Cantilever Beam with Differenti Tension and Compression Modulus. Journal of Mechanical Strength, 27(2), pp.262-267, 2005.

[13] Wu Xiao, Yang Lijun. Elastic solutions for thick wall spherical shell of bimodulous materials under uniform pressure. Journal of Hunan University of Science\&Technology(Natural Science Edition), 27(4), pp. 35-38, 2012. 